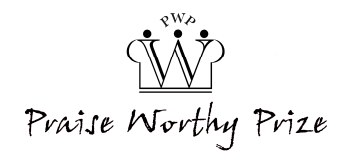

\title{
8-12 GHz pHEMT MMIC Low-Noise Amplifier for 5G and Fiber-Integrated Satellite Applications
}

\author{
Mfonobong Uko, Sunday Ekpo
}

\begin{abstract}
The fifth-generation (5G) radio access technology promises to revolutionise integrated earth-space communications applications for ubiquitous, seamless and broadband services. The assigned sub-6 GHz and millimetre-wave $5 G$ frequencies require the sensitivity of the receiver front-end subsystem(s) to detect and amplify the desired signal at a noise floor of less than -90 dBm for a cost-effective infrastructure deployment. This paper presents a broadband Monolithic Microwave Integrated Circuit (MMIC) Low-Noise Amplifier (LNA) design based on a $0.15 \mu \mathrm{m}$ gate length Indium Gallium Arsenide (InGaAs) pseudomorphic high electron mobility transistor (pHEMT) technology for $5 G$ and fiber-integrated satellite communications applications. The designed three-stage 8-12 GHz LNA implements a common-source topology. The MMIC LNA subsystem performance demonstrates an industry-leading in-band gain response of $40 \mathrm{~dB}$; a noise figure of $1.0 \mathrm{~dB}$; and a power dissipation of $43 \mathrm{~mW}$. For a constant bandwidth receiver, the sensitivity changes by approximately $1.5 \mathrm{~dB}$ over the operating satellite signal frequency. Similarly, for a variable bandwidth receiver, the sensitivity changes by approximately $1.5 \mathrm{~dB}$ over the channel bandwidth. Moreover, the sensitivity margin of the designed LNA is $40 \mathrm{~dB}$ and this holds a great promise for real-time radio access component-level reconfiguration applications. Copyright $\left.{ }^{(}\right) 2020$ The Authors.

Published by Praise Worthy Prize S.r.l. This article is open access published under the CC BY-NC-ND license (http://creativecommons.org/licenses/by-nc-nd/3.0/).
\end{abstract}

Keywords: Low-Noise Amplifier, Radio-Over-Fiber, Receiver Sensitivity, Satellite Communication

\section{Nomenclature}

$3 \mathrm{G}$

$4 \mathrm{G}$

$5 \mathrm{G}$

BER

CNR

ENP

ETRI

$f_{\mathrm{d}}$ FISCA

$f_{\text {max }}$

$f_{\mathrm{T}}$

InGaAs

LNA

LNB

LO

MDS

MER

MMIC

NF pHEMT PLL $P_{\text {r }}$

$\begin{array}{ll}\text { RATs } & \text { Radio Access Technologies } \\ \text { RoF } & \text { Radio-over-Fiber } \\ \text { SNR } & \text { Signal-to-Noise Ratio } \\ \text { UE } & \text { User Equipment } \\ \text { VCO } & \text { Voltage-Controlled Oscillator } \\ V_{\mathrm{ds}} & \text { Drain-source voltage } \\ V_{\mathrm{gs}} & \text { Gate-source voltage }\end{array}$

\section{Introduction}

The increasing global demand for broadband data, voice and video services has led to the development of advanced Radio Access Technologies (RATs) such as the 5G communication standard [1]. The 5G RAT implementation across the niche wireless communication and vertical industries/sectors is constrained by the availability of reliable physical layer devices such as the Monolithic Microwave Integrated Circuit (MMIC) Low Noise Amplifier (LNA). Integrated earth-space heterogeneous communication infrastructure requires adaptive space assets [2], [3] and advanced radio access technologies ecosystem (such as 5G). Integrated 5Gsatellite systems are required to deliver space-enabled reliable transmission [3] of ultra-low latency [4], [5], massive machine-to-machine (M2M) and broadband 
high-speed [6] data over the merged transceivertransponder channels. Data communication reliability is a function of the subsystems' adaptation [3] to the stochastic nature of the channel. Consequently, the space and the terrestrial environments are different and both pose peculiar data transmission challenges [3]. The proposed reconfigurable ubiquitous seamless communication [3] requires a judicious analysis of the user-equipment-base station-satellite link performance [7]-[9]. The system-level design considerations revolve around high teledensity communication threshold; noise and interference contributions constraints; power consumption requirement; and sensitivities of the receivers [10], [11]. Specifically, the X-band frequency is for space research, deep space operations, and environmental and military communication satellites [12].

This paper examines the relationship between the mmwave receiver sensitivity and the noise floor over the Xband for Fiber-Integrated Satellite Communication Architecture (FISCA) applications (Fig. 1).

Fig. 2 shows the X-band satellite communication transponder subsystem. It consists of the receive antenna, band-pass filter, the Low-Noise Amplifier (LNA), and the frequency synthesizer [comprising the Phase Lock Loop (PLL), Local Oscillator (LO) and VoltageControlled Oscillator (VCO)], the baseband processor for analog-to-digital conversion [13]. The LNA is a vital building block of the receiver front-end of the satellite transponder subsystem; it determines the system's overall noise temperature performance and link reliability (including antenna gain to noise temperature (G/T) specification) [8], [9].

In this paper, a $0.15 \mu \mathrm{m}$ pHEMT process is used to design a MMIC LNA for fiber-integrated 5G-satellite reception communication system applications. Section II examines the active device process technologies selection respecting the existing and emerging Radio Access Networks (RANs) standards. The broadband design procedures for the MMIC LNA are presented in Section III.

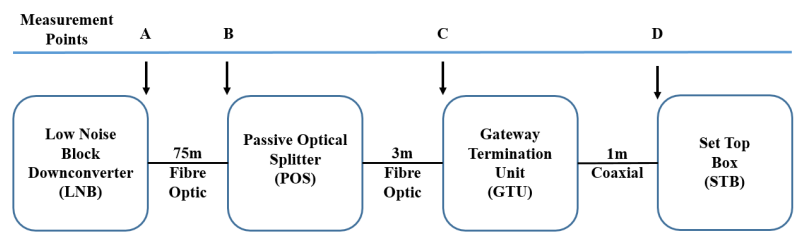

Fig. 1. Fiber-integrated satellite communication architecture

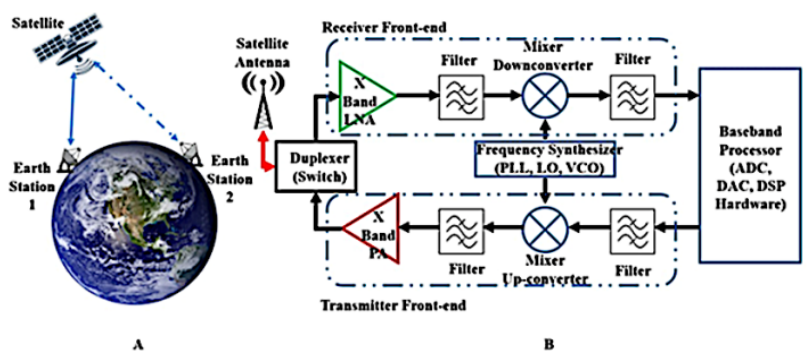

Fig. 2. An X-band satellite transponder subsystem
The pertinent design, modeling and simulation results are presented in Section IV. Section V concludes the paper.

\section{Active Device Technology Selection}

The right process technology is vital to meet the design goals and expected level of performance of an integrated transceiver-transponder system. The parametric simulation models of a foundry library is required to be scalable to allow for hardware-in-the-loop and dynamic link library components design adaptations.

Next-generation integrated 5G-satellite receivers need to provide high sensitivity and selectivity over multiband, multi-standards heterogeneous communication applications. To accomplish this, the receiver front-end LNA should be designed and optimized to give high gain and linearity over the required frequency band and process technologies including pHEMTs [14], [15] and silicon-germanium (SiGe) Heterojunction Bipolar Transistors (HBTs) [16], [17]. The pHEMT process technology offers a high power-added efficiency performance with an excellent low noise. It is most appropriate for applications (including satellite, radar and microwave radio communication systems) in which the optimization of the receiver front-end sensitivity is a critical requirement [8], [18]. The designed LNA reported in this paper uses the $0.15 \mu \mathrm{m}$ InGaAs pHEMT process technology from the WIN foundry library. The InGaAs pHEMT is known to demonstrate outstanding noise performance, lower cost and better robustness at microwave frequencies [19]. The active device exhibits a unity current gain frequency $\left(f_{T}\right)$ of $110 \mathrm{GHz}$ and a maximum oscillation frequency $\left(f_{\max }\right)$ of over $150 \mathrm{GHz}$. To understand the non-linear characteristics of the pHEMT and the operating point of the transistor, dc and RF tests/measurements over different bias conditions are carried. The active semiconductor pHEMT device modeling is shown in Fig. 3. These I-V characteristics are performed to determine the operating point for the MMIC LNA stages respecting the power consumption per stage.

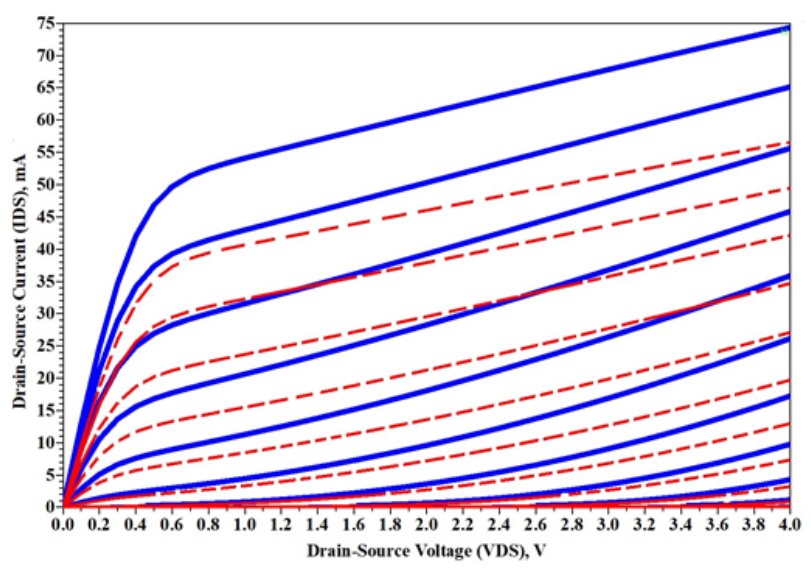

Fig. 3. I-V characteristics for a $2 \times 75$ (dash lines) and $2 \times 100$ (solid lines) $\mu \mathrm{m}$ InGaAs pHEMT 
In this research, the operating point parameters for acceptable performance of the MMIC LNA were obtained as follows: drain-source voltage $V_{d s}=2 \mathrm{~V}$; gatesource voltage $V_{g s}=-0.3 \mathrm{~V}$ and drain-source current $I_{d s}=0.021 \mathrm{~A}$. This operating point modeled the optimum low-noise bias for the X-band MMIC LNA.

Fig. 4 shows the characteristics curve for the dc transconductance, $g_{m}$, of a $2 \times 75 \mu \mathrm{m}$ InGaAs pHEMT.

The $g_{m}$ parameter determines the forward transmission gain of the MMIC LNA. It shows the relationship between the current through the output (drain) of the transistor and the voltage across the input (gate) of the transistor.

Mathematically, the transconductance of a given transistor configuration is:

$$
g_{m}=\frac{\Delta I_{d s}}{\Delta V_{g s}}
$$

From Fig. 4, the transconductance value obtained is 81 $\mathrm{mS}$ at $V_{d s}=2 \mathrm{~V}, V_{g s}=-0.3 \mathrm{~V}$ and $I_{d s}=0.021 \mathrm{~A}$.

\section{Broadband MMIC LNA Design}

A single-ended MMIC LNA is designed over the frequency range of 8-12 GHz for integrated 5G-satellite applications. The active device makes use of a scalable $0.15 \mu \mathrm{m}$ low noise InGaAs pHEMT process technology.

The most critical part of the amplifier design is the input matching network where the $50 \Omega$ input termination is converted into a complex impedance; this must be as close as possible to the optimum noise match of the transistors [20].

The choice of a process technology alongside transistor geometry is vital for amplifier characterization in terms of noise figure performance. Three distinct LNA topologies [20] are popular in terms of design and performance optimization [21]. These include: Commonsource, common-gate, and cascode LNA topologies. The common-source [20] topology gives high gain and good noise performance while the common-gate topology gives a lower power consumption rate.

At a high frequency, the common-source topology improves the stability and linearity of the amplifier, giving an excellent input impedance match with the lowest possible noise figure at the expense of overall gain.

The common-gate [20] topology also improves the stability of the amplifier and its linearity with a much higher gain and noise figure than the common-source configuration.

The cascode [20] topology improves stability, linearity and gain flatness.

However, the cascode topology degrades noise and gain performance due to increased substrate parasitics at higher frequencies. The inductive-source-degenerated common-source topology is selected as the starting point for this design for a low noise performance and the stability of the first-stage transistor.

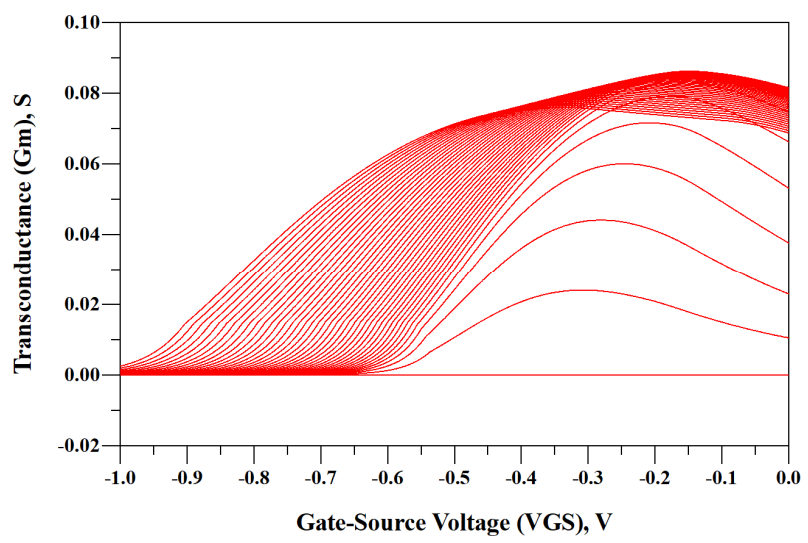

Fig. 4. Transconductance responses for a $2 \times 75 \mu \mathrm{m}$ InGaAs pHEMT

\section{III.1. Noise Characterization}

Noise is an undesired signal added by system components. It is an important figure-of-merit in microwave design that degrades the performance of transmitted signals. For integrated 5G-satellite systems, the noise figure is determined using the performance metrics of the low-noise amplifier, mixers, and oscillators at the receiver front-end.

For a multistage amplifier system, the overall noise figure is expressed as:

$$
F=F_{1}+\frac{F_{2}-1}{G_{A 1}}+\frac{F_{3}-1}{G_{A 1} G_{A 2}}+\cdots+\frac{F_{n}-1}{G_{A 1} G_{A 2} . G_{A(n-1)}}
$$

\section{III.2. X-Band LNA Design}

The X-Band LNA design spans $8-12 \mathrm{GHz}$ of the electro-magnetic spectrum. A three-stage X-Band low noise amplifier is designed using a $0.15 \mu \mathrm{m}$ low noise InGaAs pseudomorphic high electron mobility transistor process technology. The various design parameters (forward transmission gain, minimum noise figure, $\mathrm{S}$ Parameter extraction, noise resistance, input and output isolations) were obtained at a bias gate-source voltage, $V_{g s}=-0.3 \mathrm{~V}$ and drain-source voltage, $V_{d s}=2 \mathrm{~V}$. This bias point is chosen to reduce the current dissipation and maintain an acceptable performance level of the LNA. In addition, this common bias network is used to ensure equal distribution of current to all stages. The resonant frequency for this design was $f_{\mathrm{d}}=10 \mathrm{GHz}$. The threestage LNA structure is shown in Fig. 5.

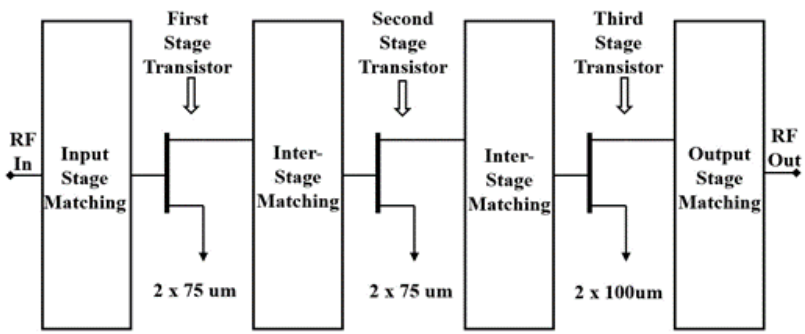

Fig. 5. Three-stage LNA structure 
Stage one: The first stage is made up of a $2 \times 75 \mu \mathrm{m}$ transistor with source inductive feedback for stabilization. The inductive series feedback in the first stage drives the optimum noise match closer to the optimum gain match, presenting the optimum match, Topt over the operating frequency to the gate of the first stage transistor with a $50 \Omega$ input source. A series LC network is utilized for the input match.

Stage two: The second stage is made up of a $2 \times 75 \mu \mathrm{m}$ transistor. An inter-stage matching network is designed to transform the output impedance of stage one transistor to the input impedance of the second stage transistor for maximum stable gain and adequate linearity.

Stage three: The third stage is made up of a $2 \times 100 \mu \mathrm{m}$ transistor. An inter-stage matching network is designed to transform the output impedance of stage two transistor to the input impedance of the third stage transistor for maximum stable gain. Parallel feedback between the input and output of the third stage is introduced for gain flatness.

Fig. 6 shows the schematic of the X-band MMIC LNA, designed to achieve a low noise figure. C1 and $\mathrm{C} 11$ act as dc blocks, preventing dc voltage from passing through the RF path. $\mathrm{C} 2, \mathrm{C} 4, \mathrm{C} 5, \mathrm{C} 7, \mathrm{C} 8$ and $\mathrm{C} 10$ are decoupling capacitors in parallel to the supply voltage.

They short any RF signal from leaking into the voltage supply path. R1, R3, R5 are high resistive loads that prevent RF leakage into the voltage supply path. The series RC network made of C9 and R6 is used in the third stage as a series feedback for gain flatness. C1 and L1 combine for the first stage optimum noise input-matching network. The optimization of the first stage for minimum noise figure with sufficient gain is required. Inductor L6 and capacitor $\mathrm{C} 11$ constitute the output-matching network for the single-ended MMIC LNA topology design. The pHEMTs devices are M1, M2 and M3 (Fig. 6). Components including dc blocking capacitor and inter-stage matching inductor are between the LNA stages for maximum power transfer.

Between the first stage, second stage and third stage active devices of the LNA, a dc blocking capacitor and inter-stage matching inductor is incorporated for maximum power transfer.

\section{III.3. X-Band Receiver Sensitivity Modeling}

The ambitious goals of 5G wireless technology can only be accomplished through an innovative assessment of the changes needed in the RF receiver system architecture to achieve higher data rates at the RF, microwave and millimetre-wave frequencies. Moreover, heat generation is a key challenge facing the RF components developers for $5 \mathrm{G}$ applications due to the massive multiple input multiple output systems and big data processing. Hence, there is a need for a judicious investigation and multiphysics characterisation (including EM and thermal simulations and validations) of active semiconductor devices process technologies for the RF receiver subsystem design for $5 \mathrm{G}$ communication applications. A new RF transceiver subsystem design paradigm for a sustainable and reliable broadband performance is needed. The authors propose a reconfigurable high-frequency low-noise amplifier for receiver sensitivity improvement for channel-aware 5G and satellite communications applications.

The sensitivity of a receiver correlates directly with its $N F$. For instance, if the $N F$ of a receiver is reduced by 1 $\mathrm{dB}$, the receiver gains $1 \mathrm{~dB}$ of sensitivity. The reception and transmission of multiple satellite transponder signals are sustainably possible through the fiber-integrated reception and transmission technology. Hence, to model the sensitivity of a typical fiber-integrated X-band receiver, the performance metrics of the front-end fiber Low-Noise Block (LNB) subsystem constitute the key design considerations.

The scope of this research is to enhance the operational and spectrum efficiencies of high data rate communication systems that incorporate radio-over-fiber (RoF) for satellite downstream and upstream applications. The signal-to-noise ratio (SNR) in $\mathrm{dB}$ of a receiver is given by:

$$
S N R=P_{r}-M D S
$$

where $P_{\mathrm{r}}=$ received power in $\mathrm{dBm}$; and $M D S=$ minimum detectable signal or receiver sensitivity in $\mathrm{dBm}$.

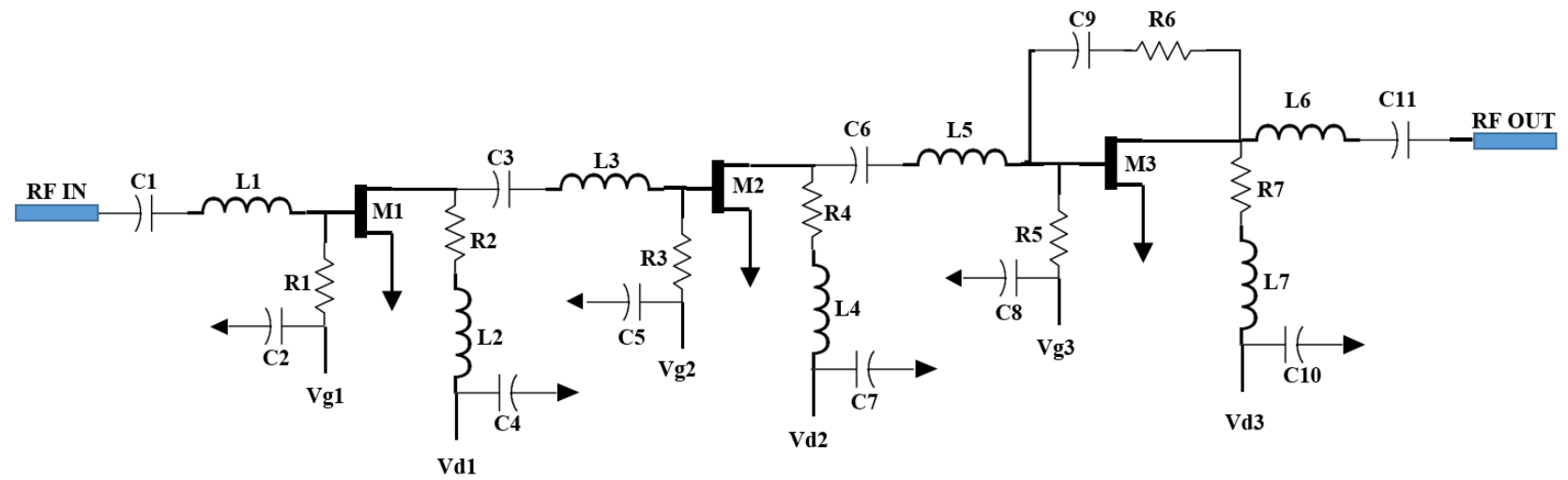

Fig. 6. A three-stage 8-12 GHz MMIC LNA design schematic 
The reference receiver sensitivity estimating relationship, $S$, in $\mathrm{dBm}$ is given by:

$$
S=10\left(\log _{10}^{K T B}\right)+N F+C N R
$$

where $K$ is the Boltzmann's constant $(=-228$ $\mathrm{dBW} /(\mathrm{kHz}) ; T$, the thermodynamic temperature of the receiver in Kelvin; $B$, the channel bandwidth (resolution) of the signal; $N F$, the noise figure of the receiver in $\mathrm{dB}$; and $\mathrm{CNR}$, the carrier-to-noise ratio in $\mathrm{dB}$ and thermal noise floor $=10\left(\log _{10}{ }^{(k T)}\right)$. The effective noise power, $E N P=10\left(\log _{10}{ }^{(\mathrm{kT} \mathrm{B})}\right)$.

From (4), the reference receiver sensitivity estimating relationship $S(f)$, in $\mathrm{dBm}$ is given by:

$$
S(f)=\frac{1}{2}\left[10\left(\log _{10}^{K T B}\right)+N F+P_{r}\right]
$$

Equation (5) indicates that the sensitivity of a receiver is largely dependent upon the bandwidth (all other parameters being within controllable boundaries). Where the noise figure of the receiver front-end subsystem changes over the operating bandwidth and with time, the relevant estimating relationship is utilized for circuit topology-specific model validation. For the designed MMIC X-band LNA, the parametric-based mathematical model for the noise figure, $N F$, is given by:

$$
N F=0.0055 f^{2}+0.0554 f+1.0505
$$

where $f$ is the operating frequency in GHz. The model represented by (6) has a coefficient of determination of 0.98 and frequency boundary conditions of $f_{\text {lower }}=8 \mathrm{GHz}$ and $f_{\text {upper }}=12 \mathrm{GHz}$.

From (5) and (6), the reference receiver sensitivity estimating relationship, $S(f)$, in $\mathrm{dBm}$ is given by:

$$
\begin{aligned}
S(f) & =\frac{1}{2}\left[10\left(\log _{10}^{K T B}\right)+0.0055 f^{2}+\right. \\
& \left.+0.0554 f+1.0505+P_{r}\right]
\end{aligned}
$$

From (7), $S(f)$, in $\mathrm{dBm}$ is given by:

$$
\begin{aligned}
S(f)= & \frac{1}{2}\left[-174+10\left(\log _{10}^{B}\right)+0.0055 f^{2}\right. \\
& \left.+0.0554 f+1.0505+P_{r}\right]
\end{aligned}
$$

Taking the derivative of equation (4) as a function of the receiver bandwidth with receiver constraints application yields thus:

$$
\frac{\delta S}{\delta B}=\frac{10}{B \ln (10)}
$$

Equation (7) shows that a reconfigurable front-end receiver (LNA) subsystem [8], [22] would be an ideal device architecture for fiber-integrated satellite communications application to achieve and sustain constant in-band noise figure regime for multi-frequency advanced RAT network operations. The need for spectral efficiency for the terrestrial- and space-borne User Equipment (UE) poses a key challenge for satellite receiver sensitivity where real-time dynamic frequency assignments and multi-band, multi-standards radio communication must be maintained for massive highspeed systems.

This paper proposes a differential deduction of the receiver sensitivity based on the process technology of the front-end LNA subsystem responses. Constant CNR and negligible in-band noise figure are required to ensure a reliable deterministic radio communication for FiberIntegrated Satellite Communication Application (FISCA). Reconfigurable and/or adaptive RF, microwave and millimeter-wave receiver front-end subsystems [8] are required for a FISCA application [30].

\section{Results and Discussion}

\section{IV.1. X-Band LNA Analysis}

The LNA S-parameters are shown in Fig. 7. It is observed that the output and input return losses are less than $-10 \mathrm{~dB}$ for the entire band. The output return loss is designed to be less than $-22 \mathrm{~dB}$.

The gain shown in Fig. 8 is $40 \mathrm{~dB}$ at the centre design frequency. The in-band ripple factor across the required operating frequency band is $1 \mathrm{~dB}$.

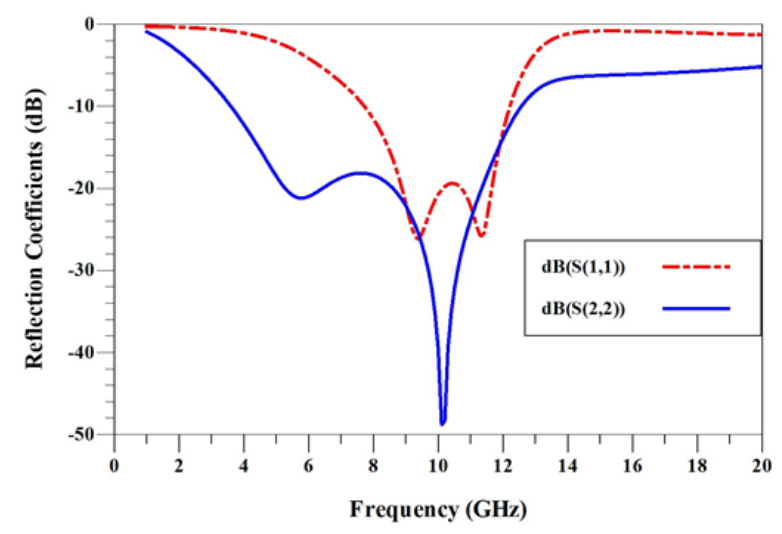

Fig. 7. Input and Output reflection coefficient of the X-band MMIC LNA circuit

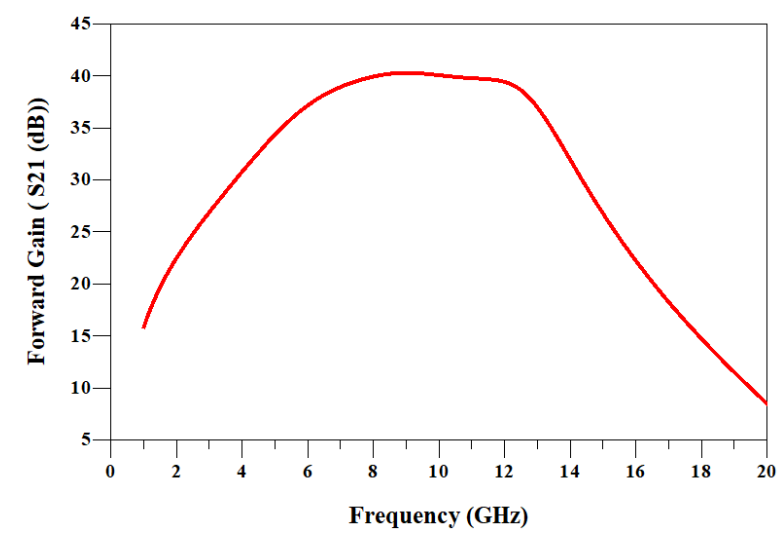

Fig. 8. Gain of the X-band MMIC LNA circuit 
The isolation loss is shown to be below $-50 \mathrm{~dB}$ (Fig. 9). The minimum noise at the resonant frequency of 10 $\mathrm{GHz}$ was $0.9 \mathrm{~dB}$ (Fig. 10). The LNA is stable across the entire band up to cut-off frequency as shown in Fig. 11.

A summary of the designed LNA performances is given in Table I. Fig. 12 shows the 1-dB compression point of the LNA. The input and output powers at 1-dB compression points are found to be $-30 \mathrm{dBm}$ and $9 \mathrm{dBm}$ at $10 \mathrm{GHz}$ respectively. Beyond these points, the amplifier goes into compression and becomes saturated.

Any further increase in the input power leads to no further output power increase. The amplifier response becomes non-linear and produces signal distortion, harmonics, and inter-modulation products beyond this compression point. Table II shows a comparison between related designs with the simulated LNA performance.

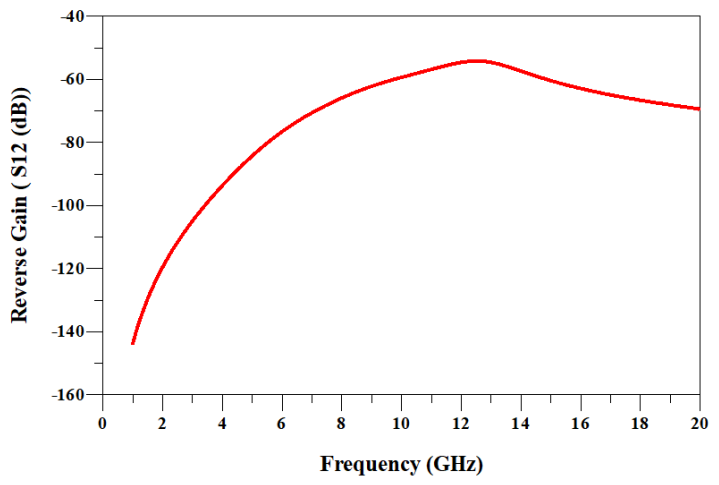

Fig. 9. Isolation of the X-band MMIC LNA circuit

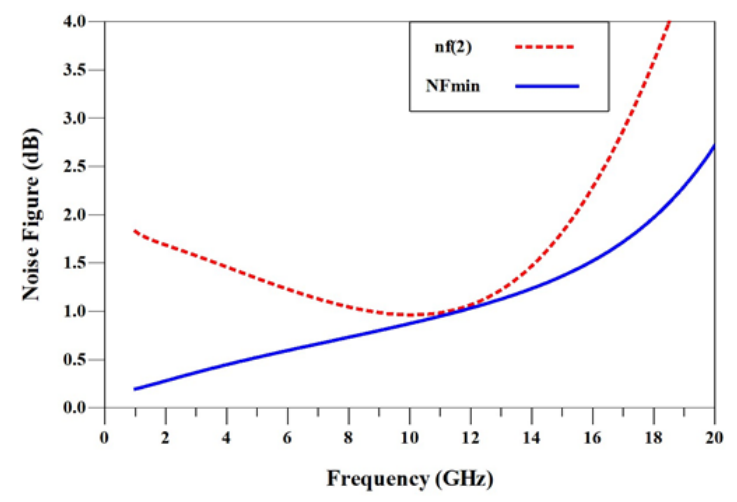

Fig. 10. Noise Figure of the X-band MMIC LNA circuit

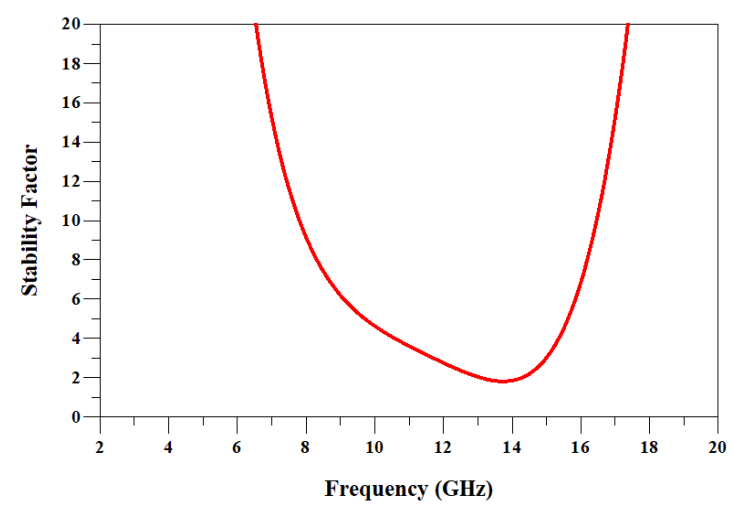

Fig. 11. Stability Factor of the X-band MMIC LNA circuit

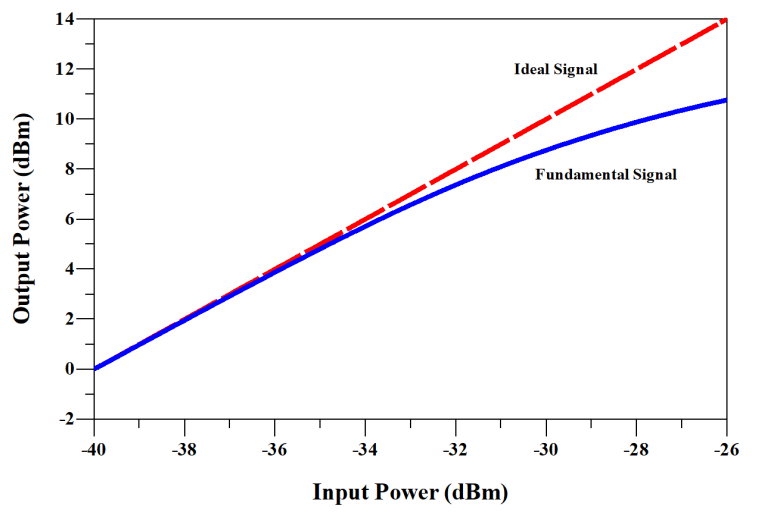

Fig. 12. $1 \mathrm{~dB}$ compression point of designed X-Band LNA

TABLE I

X-BAND LNA REQUIREMENTS AND PERFORMANCE AT $10 \mathrm{GHz}$ DESIGN FREQUENCY

\begin{tabular}{ccc}
\hline \hline Design Parameter & Requirement & Performance \\
\hline $\mathrm{S}_{11}(\mathrm{~dB})$ & -10 & -21 \\
$\mathrm{~S}_{12}(\mathrm{~dB})$ & -40 & -60 \\
$\mathrm{~S}_{21}(\mathrm{~dB})$ & 25 & 40 \\
$\mathrm{~S}_{22}(\mathrm{~dB})$ & -10 & -40 \\
$\mathrm{NF}(\mathrm{dB})$ & $<2$ & 0.9 \\
$\mathrm{~K}$ & $>2$ & 5 \\
In-band Ripple $(\mathrm{dB})$ & 3 & 1 \\
\hline \hline
\end{tabular}

\section{IV.2. FIS X-Band Receiver Sensitivity Simulation}

In order to characterize and analyse the sensitivity response of the reported designed broadband $8-12 \mathrm{GHz}$ InGaAs pHEMT MMIC LNA for fiber-integrated satellite LNB, a simulation of the performance metrics for a typical X-band receiver was carried out. The simulation parameters that were applied in (3), (4), (8) and (9) are thus: $C N R$ (measured) $=17 \mathrm{~dB} ; N F=0.9 \mathrm{~dB}$ (from the presented designed 8-12 GHz MMIC LNA); $f_{\mathrm{c}}$ $=9.8 \mathrm{GHz}$; satellite channel bandwidth (resolution), $\mathrm{B}=$ $50 \mathrm{MHz}$ (typical); measured received power, $P_{\mathrm{r}}=$ $33 \mathrm{dBm}$. Figure 13 illustrates the $\mathrm{X}$-band receiver sensitivity and noise floor responses over the variable channel bandwidths of the fiber LNB. A reconfigurable $5 \mathrm{G}$ receiver with the capability to adapt the channel bandwidth to the on-demand duplex signal transmission and reception constraints can sup-port optimal real-time receiver sensitivity threshold. For a constant channel bandwidth (Fig. 14), the $50 \mathrm{MHz}$ margin yields the best receiver sensitivity threshold [8] over the X-band operating frequency. This appears to justify the choice of the $50 \mathrm{MHz}$ bandwidth for satellite video signals transmission [22], [30]. During a MMIC LNA characterization, the allowable temperature drift is $23 \pm 1$ ${ }^{\circ} \mathrm{C}$; some equipment allow for up to $3{ }^{\circ} \mathrm{C}$ drift about the ambient temperature. For any given fabricated MMIC LNA that is characterized, 0.1 to $0.2 \mathrm{~dB}$ is the allowable loss margin for linear (S-parameters) measurements. A loss of above $1 \mathrm{~dB}$ is unacceptable. The finding implies that there is a need to enhance the receiver sensitivity by performing a component- to subsystem-levels reconfiguration in near real-time. 
TABLE II

COMPARISON OF SiMULATED LNA AT X BAND FREQUENCIES

\begin{tabular}{|c|c|c|c|c|c|c|}
\hline Ref. & Process & No. of Stages & Freq.(GHz) & Gain (dB) & Noise $(\mathrm{dB})$ & Power $(\mathrm{mW})$ \\
\hline [25] & $0.65 \mu \mathrm{m} \mathrm{CMOS}$ & 2-Stage MMIC & $9.35-10.65$ & 27.8 & 1.8 & 4.68 \\
\hline [26] & $0.25 \mu \mathrm{m} \mathrm{GaN}$ & 4-Stage MMIC & $8-12$ & 20.2 & 4.3 & 100 \\
\hline [27] & $0.13 \mu \mathrm{m}$ SiGe BiCMOS & 3-Stage MMIC & $8-18$ & $50-53$ & $6.7-7.8$ & 180 \\
\hline [28] & $0.7 \mu \mathrm{m}$ GaAs mHEMT & 3-Stage MMIC & $7-11$ & 30 & 1 & 62.2 \\
\hline [29] & $0.1 \mu \mathrm{m}$ GaAs mHEMT & 3-Stage MMIC & $4-12$ & 31.5 & 1.31 & 8 \\
\hline [30] & $0.1 \mu \mathrm{m}$ InP HEMT & 3-Stage MMIC & $0.3-14$ & 40 & 2.73 & 12 \\
\hline [31] & $0.13 \mu \mathrm{m}$ SiGe BiCMOS & 3-Stage MMIC & $6-12$ & 21 & 1.7 & 100 \\
\hline This Work & $0.15 \mu \mathrm{m}$ InGaAs pHEMT & 3-Stage MMIC & $8-12$ & 40 & 0.9 & 43 \\
\hline
\end{tabular}

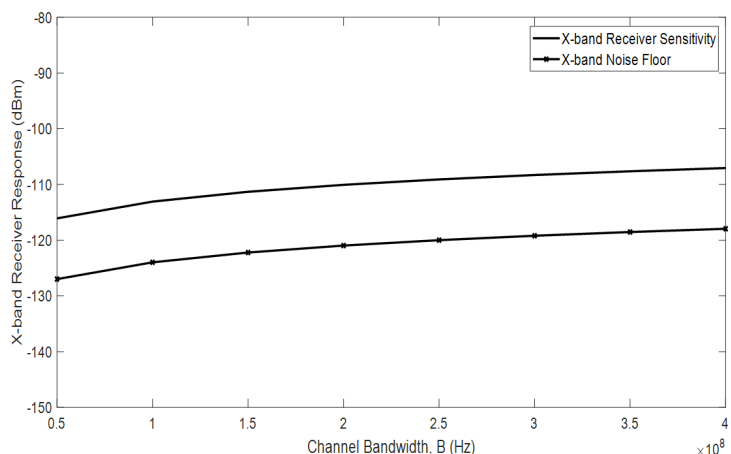

Fig. 13. Fibre-Integrated Receiver Sensitivity Response

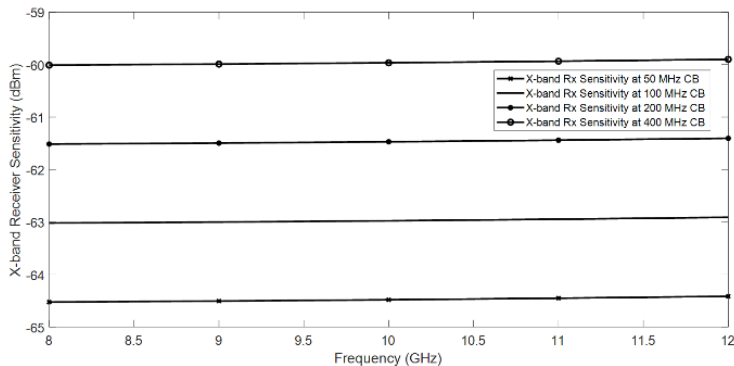

Fig. 14. X-band Receiver Sensitivity at a Constant Channel Bandwidth

The reported three-stage single-ended X-band MMIC LNA design uses the common-source configuration (CSC) for low-noise performance and moderate bandwidth.

The series LC impedance matching network and circuit design technique enhances the gain and stability. Parallel feedback in stage three improves the gain flatness of the forward transmission response. This finding further strengthens the need for a reconfigurable receiver to cater for the massive $5 \mathrm{G}$ UE and base station transceivers operational requirements.

\section{IV.3. Validation of the X-Band LNA for FISCA}

The presented findings in this paper have been independently verified with measurement-based validation. Fig. 15 shows the responses of the measured $\mathrm{CNR}$ and the designed $\mathrm{X}$-band sensitivity as a function of frequency.

The weather parameters of the FISCA measurement system are stated in Table III. Bit Error Ratio (BER) and Modulation Error Ratio (MER) [30] provide a good indication of a satellite signal quality.

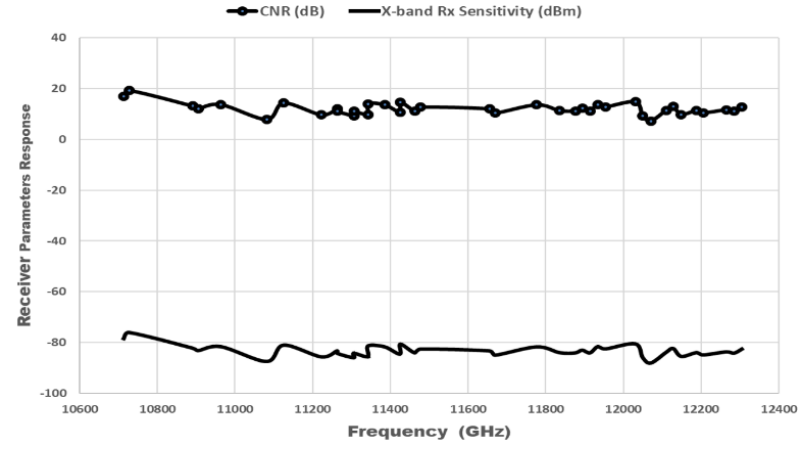

Fig. 15. Measured FISCA CNR and X-band Sensitivity Responses

TABLE III

Clear Hot DAy WeATHER DATA

\begin{tabular}{cc}
\hline \hline Parameter & Value \\
\hline Time & $14: 00$ \\
Total Cloud Cover (Oktas) & 0 \\
Visibility (meters) & 45000 \\
Pressure (hPa) & 1015.8 \\
Air Temperature ( C) & 22.2 \\
Wind Speed (knots) & 4 \\
Relative Humidity (\%) & 48.4 \\
Sunshine Hours (\%) & 100 \\
\hline \hline
\end{tabular}

The sensitivity of the receiver is an important factor to realize a good BER value $\left(10^{9}\right)$. From Fig. 15 , the average sensitivity of the designed $\mathrm{X}$-band receiver is -83 $\mathrm{dBm}$.

This defines the required signal strength at the receiver input and serves as the first step in satellite link budget design.

Remotely reconfiguring the satellite receiver bandwidth by an order of 10 (i.e., $5 \mathrm{kHz}$ ) introduces a sensitivity margin of $40 \mathrm{~dB}$.

The single-ended MMIC LNA has been the preferred architecture for most of the published simulated and measured low-noise communications applications.

The prevailing active device configuration for low noise applications is common-source or common-gate [8].

An et al. have published 1.5 to $2.5 \mathrm{GHz}$ MMIC LNA design with measured results differing from the simulation responses by less than $20 \%$ for both the gain and the noise figure of the amplifier [18]. The process variations are deemed to cause this difference. Moreover, the $0.15 \mu \mathrm{m} \mathrm{GaAs}$-based pHEMT process [20] has been utilized to characterize MMIC LNAs over the mm-wave frequencies [8]. The variations between the simulated and the measured gain and noise figure responses are approximately $1 \mathrm{~dB}$. 


\section{Conclusion}

A three-stage X-band MMIC low-noise amplifier has been designed using the common source topology. The pHEMT process technology was utilized, and the performance metrics of the fiber low-noise block frontend satisfies the requirements for fiber-integrated satellite communication applications. The amplifier stability up to the cut-off frequency is greater than 2 and meets the fabrication defects standards for zero oscillation performance. The measured receiver parameters reveal an average carrier-to-noise ratio of 12 $\mathrm{dB}$; and sensitivities of $-84 \mathrm{dBm}$ and $-124 \mathrm{dBm}$ at bandwidths of $50 \mathrm{MHz}$ and $5 \mathrm{kHz}$ respectively. The Xband sensitivity response to channel bandwidth variations is bandwidth and operating frequencydependent, with a low noise figure of $0.9 \mathrm{~dB}$. Hence, this reduces system integration complexity, deployment and cost of user and base station equipment. The reported findings in this paper have revealed the huge communication improvements that integrated advanced radio access technologies (such as 5G) and satellite communication would add to the global connectivity infrastructure and services through capability-based reconfigurable radio communication subsystems. The future research direction of this novel work will consider the integration of the LNA with a monolithic reconfigurable switch for multi-frequency adaptive capabilities.

\section{References}

[1] Idowu-Bismark, O., Okokpujie, K., Husbands, R., Adedokun, M., 5G Wireless Communication Network Architecture and Its Key Enabling Technologies, (2019) International Review of Aerospace Engineering (IREASE), 12 (2), pp. 70-82. doi:https://doi.org/10.15866/irease.v12i2.15461

[2] J. Fang, C. Zhang, F. W. Singor, and J. A. Abraham, "A Broadband CMOS RF Front End for Direct Sampling Satellite Receivers," IEEE Journal of Solid-State Circuits, vol. 54, no. 8, pp. 2140-2148, Aug 2019.

[3] Ekpo, S., Thermal Subsystem Operational Times Analysis for Ubiquitous Small Satellites Relay in LEO, (2018) International Review of Aerospace Engineering (IREASE), 11 (2), pp. 48-57. doi:https://doi.org/10.15866/irease.v11i2.13663

[4] Y. Xu, G. Wang, S. Wei, E. Blasch, K. Pham, and G. Chen, "High-throughput, Cyber-Secure Multiuser Superposition Covert Avionics System," IEEE Aerosp. Electron. Syst., vol. 33, no. 2, pp. 4-15, February 2018.

[5] L. Bai, L. Zhu, X. Zhang, W. Zhang, and Q. Yu, "Multi-Satellite Relay Transmission in 5G: Concepts, Techniques, and Challenges," IEEE Net., vol. 32, no. 5, pp. 38-44, September 2018.

[6] J. Lin, "Synchronization Requirements for 5G: An Overview of Standards and Specifications for Cellular Networks," IEEE Veh. Technol., vol. 13, no. 3, pp. 91- 99, Sept 2018.

[7] T. Do-Duy and M. A. Vazquez-Castro, "Network Coding Function for Converged Satellite-Cloud Networks," IEEE Aerosp. Electron. Syst., pp. 1-1, 2019.

[8] S. C. Ekpo and D. George, "Impact of Noise Figure on a Satellite Link Performance," IEEE Commun. Letters, vol. 15, no. 9, pp. 977-979, September 2011.

[9] W. Lee, N. Luhrs, K. Isbell, C. Oliphant-Jerry, P. Morris, and Y. Hong, "Cavity-Backed Archimedean Spiral Antenna with Conical Perturbations for 3U CubeSat Applications [Education Corner]," IEEE Antennas Propag., vol. 60, no. 6, pp. 102-109, Dec 2018.
[10] K. Kaneko, H. Nishiyama, N. Kato, A. Miura, and M. Toyoshima, "Construction of a Flexibility Analysis Model for Flexible HighThroughput Satellite Communication Systems With a Digital Channelizer," IEEE Trans. Veh. Technol., vol. 67, no. 3, pp. 2097-2107, March 2018.

[11] T. Delamotte and A. Knopp, "Smart Diversity Through MIMO Satellite Q/V-Band Feeder Links," IEEE Aerosp. Electron. Syst., pp. 1-1, 2019.

[12] Bendoukha, S., Tapia, I., Okuyama, K., Cho, M., An Experimental and Theoretical Study of Spatial Langmuir Probe Plasma System for a Small Lean Satellite Called Ten-Koh, (2019) International Review of Aerospace Engineering (IREASE), 12 (3), pp. 131-140. doi:https://doi.org/10.15866/irease.v12i3.15927

[13] Kabirov, V., Semenov, V., Shinyakov, Y., A Digital Control System for the Power Conditioning Unit of Spacecraft, (2019) International Review of Aerospace Engineering (IREASE), 12 (1), pp. 26-34. doi:https://doi.org/10.15866/irease.v12i1.15573

[14] C. V. N. Rao, D. K. Ghodgaonkar, and N. Sharma, "GaAs MMIC Low Noise Amplifier With Integrated High Power Absorptive Receive Protection Switch," IEEE Microw. Wireless Compon. Lett., vol. 28, no. 12, pp. 1128-1130, Dec 2018.

[15] T. Kulatunga, L. Belostotski, and J. W. Haslett, "400to-800-MHz GaAs pHEMT-Based Wideband LNA for Radio-Astronomy Antenna-Array Feed," IEEE Microw. Wireless Compon. Lett., vol. 28, no. 10, pp. 909-911, Oct 2018.

[16] J. D. Cressler, "SiGe HBT technology: a new contender for Sibased RF and microwave circuit applications," IEEE Trans. Microw. Theory Techn., vol. 46, no. 5, pp. 572-589, May 1998.

[17] I. Song, A. S. Cardoso, H. Ying, M. Cho, and J. D. Cressler, "Cryogenic Characterization of RF Low-Noise Amplifiers Utilizing Inverse-Mode SiGe HBTs for Extreme Environment Applications," IEEE Trans. Device Mater. Rel., vol. 18, no. 4, pp. 613-619, Dec 2018.

[18] A. Dan, R. Eung-Ho, R. Jin-Koo, and K. Sam-Dong, "Design and fabrication of a wideband MMIC Low Noise Amplifier using Qmatching," Journal of The Korean Physical Society - J KOREAN PHYS SOC, vol. 37, 122000.

[19] K. W. Kobayashi, "Linearized Darlington Cascode Amplifier Employing GaAs PHEMT and GaN HEMT Technologies," IEEE J. of Solid-State Circuits, vol. 42, no. 10, pp. 2116-2122, Oct 2007.

[20] S. Ekpo, R. Kharel, and M. Uko, "A Broadband LNA Design in Common-Source Configuration for Reconfigurable Multistandards Multi-bands Communications," in Proc. 2018 ARMMS $R F$ and Microwave Conference, April 2018, pp. 1-10.

[21] T. Das, "Practical Considerations for Low Noise Amplifier Design," Freescale Semiconductor, 2013.

[22] Ekpo, S., Adebisi, B. and Wells, A., "Regulated-element Frost Beamformer for Vehicular Multimedia Sound Enhancement and Noise Reduction Applications," IEEE Access Journal, Vol. 5, pp. 27254-27262, Dec 2017.

[23] M. R. Nikbakhsh, E. Abiri, H. Ghasemian, and M. R. Salehi, "Two-stage current-reused variable-gain lownoise amplifier for $\mathrm{X}$-band receivers in $65 \mathrm{~nm}$ complementary metal oxide semiconductor technology," IET Circuits, Devices Systems, vol. 12, no. 5, pp. 630-637, 2018.

[24] D. Kim, D. Lee, S. Sim, L. Jeon, and S. Hong, "An XBand Switchless Bidirectional GaN MMIC Amplifier for Phased Array Systems," IEEE Microw. Wireless Compon. Lett., vol. 24, no. 12, pp. 878-880, Dec 2014.

[25] D. Ma, F. F. Dai, R. C. Jaeger, and J. D. Irwin, "An Xand KuBand Wideband Recursive Receiver MMIC With Gain-Reuse," IEEE J. of Solid-State Circuits, vol. 46, no. 3, pp. 562-571, March 2011.

[26] S. Bhaumik and D. Kettle, "Broadband X-band low noise amplifier based on $70 \mathrm{~nm}$ GaAs metamorphic high electron mobility transistor technology for deep space and satellite communication networks and oscillation issues," IET Microwaves, Antennas Propagation, vol. 4, no. 9, pp. 1208-1215, Sep. 2010.

[27] B. A. A. et al, "4-12 and 25-34 GHz Cryogenic mHEMT MMIC Low-Noise Amplifiers," IEEE Trans. Microw. Theory Techn., 
vol. 60, no. 12, pp. 4080-4088, Dec 2012.

[28] E. C. et al, "0.3-14 and 16-28 GHz Wide-Bandwidth Cryogenic MMIC Low-Noise Amplifiers," IEEE Trans. Microw. Theory Techn., vol. 66, no. 11, pp. 4860-4869, Nov 2018.

[29] M. Davulcu, C. Caliskan, I. Kalyoncu, and Y. Gurbuz, "An XBand SiGe BiCMOS Triple-Cascode LNA With Boosted Gain and P1dB," IEEE Trans. Circuits Syst., II, Exp. Briefs, vol. 65, no. 8, pp. 994-998, Aug 2018.

[30] S. C. Ekpo, "Parametric System Engineering Analysis of Capability-Based Small Satellite Missions," IEEE Syst. J., pp. 27254-27262, 2019.

\section{Authors' information}

Communication and Space Systems Engineering Team, Department of Engineering, Faculty of Science and Engineering, Manchester Metropolitan University, Manchester, M1 5GD, UK.

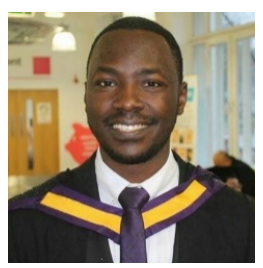

Mfonobong Uko received his B.Eng. degree in Electrical/Electronic Engineering from University of Uyo, Nigeria and MSc in Communication Engineering from The University of Manchester, Uk. He is a $\mathrm{PhD}$ candidate in Communication Engineering at the Manchester Metropolitan University, UK. His research interest are adaptive satellite system design; multi- physics design, and modelling of RF, microwave, millimetre-wave, and optical transceivers; internet of things sensors characterization; multi-objective system engineering; and complex systems optimization.

E-mail: $\underline{\text { m.uko@mmu.ac.uk }}$

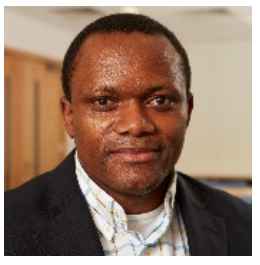

Sunday C. Ekpo obtained the MSc. Degree in Communication Engineering from the University of Manchester, Manchester, UK in September 2008 and proceeded for his Ph.D. degree in Electrical and Electronic Engineering at the same institution. He specialises in highly adaptive satellite system de- sign; multi-physics design, and modelling of $\mathrm{RF}$, microwave, millimetre-wave, and optical transceivers; internet of things sensors characterization; multi- objective system engineering; and complex systems optimization. He holds a PGC. in Academic Practice and MA. in Higher Education. Dr Ekpo is a Senior Lecturer in Electrical \& Electronic Engineering; leads the Communicationa and Space Systems Engineering research team at the Manchester Met University, UK; Chartered Engineer; and Fellow of the Higher Education Academy (UK). He is a member of the Institution of Engineering and Technology, and the American Institute of Aeronautics and Astronautics.

E-mails: scekpo@ieee.org

S.Ekpo@mmu.ac.uk 